Portland State University

PDXScholar

Electrical and Computer Engineering Faculty

Publications and Presentations

$12-1-1986$

\title{
Metal Waveguide Modeling of Tapered Quadratic Index Media
}

Lee W. Casperson

Portland State University

Bahram Zandi

Portland State University

Follow this and additional works at: https://pdxscholar.library.pdx.edu/ece_fac

Part of the Electrical and Computer Engineering Commons

Let us know how access to this document benefits you.

\section{Citation Details}

Lee W. Casperson, Bahram Zandi. Metal waveguide modeling of tapered quadratic index media. Applied Optics, Volume 25, Number 23 (December 1986), pp. 4417-4420.

This Article is brought to you for free and open access. It has been accepted for inclusion in Electrical and Computer Engineering Faculty Publications and Presentations by an authorized administrator of PDXScholar. Please contact us if we can make this document more accessible: pdxscholar@pdx.edu. 


\title{
Metal waveguide modeling of tapered quadratic index media
}

\author{
Lee W. Casperson and Bahram Zandi
}

The basic electromagnetic properties of tapered graded index fibers are equivalent to the properties of a class of concave metallic waveguides. Although useful in their own right, such metal waveguides also provide a straightforward and inexpensive means for studying the characteristics of more complicated tapered media.

\section{Introduction}

The idea that an electromagnetic wave could be guided by a cylinder having a continuous radial profile of the index of refraction has long been known. ${ }^{1}$ With newer materials and fabrication techniques, such graded index fibers are attracting increased attention because of their improved bandwidth and image transmission properties. Tapered graded index waveguides are also of interest as possible waveguide couplers, power concentrators, and image size reducers. Other variations of the index profile along the propagation direction may also be introduced intentionally or accidentally in the fiber manufacturing process. The subject of beam propagation in such $z$-dependent media has been briefly reviewed, and new theoretical solutions are discussed in Refs. 2-4.

The experimental study of $z$-dependent graded index materials is typically much more difficult than the corresponding theoretical calculations. The fabrication of well-characterized quadratic index media is not an easy process, and further difficulties may arise as one introduces variations along the propagation direction. Even when such a waveguide has been constructed, measurement of the parameters of a microscopic propagating beam may be difficult. As a result, there have been few significant comparisons between theoretical models for $z$-dependent graded index media and experimental data.

One purpose of this study is to suggest an alternative approach to the experimental demonstration and verification of graded index waveguide models. As just

The authors are with Portland State University, Department of Electrical Engineering, Portland, Oregon 97207.

Received 18 April 1986.

0003-6935/86/234417-04\$02.00/0.

(C) 1986 Optical Society of America. noted, a principal difficulty with most practical graded index waveguides is their extremely small size, and one is led to seek larger waveguide models that might be more easily fabricated and studied. However, such models can only be useful if one uses waves of much larger wavelength or media having much weaker focusing properties. The models discussed here are based on the optical equivalences between small tapered quadratic index waveguides and larger and more easily studied concave metallic waveguides.

A few years ago it was demonstrated that effective light guiding could be achieved using a single curved surface rather than a fully enclosed waveguide, ${ }^{5-9}$ and this behavior is similar to the acoustic whispering gallery guiding studied by Rayleigh. ${ }^{10,11}$ That initial light guiding work was followed by several studies of beam propagation in concave curved metallic waveguides. ${ }^{12-15}$ It was found that the eigenmodes of the waveguides involve Hermite-Gaussian functions across the surface of the guide and Airy functions perpendicular to the surface. These Airy-HermiteGaussian surface modes are a high-order limit of the Bessel-Hermite-Gaussian modes that had earlier been derived for radial mode laser resonators. ${ }^{16-19}$ More general arrangements include off-axis and bouncing modes in waveguides that may in general be shaped into a helix or which may have curvature parameters that vary with propagation distance. These waveguides are intrinsically interesting because of their fabricational simplicity and their ability to transmit high power beams of arbitrary wavelength. They have also been the basis for glass ${ }^{20}$ and metal ${ }^{21,22}$ waveguide lasers. For present purposes the most important result of the previous studies is that the equations governing the off-axis Hermite-Gaussian mode functions of a concave curved metallic waveguide are the same as the mode equations of a $z$-dependent quadratic index waveguide. Thus one can use metal waveguides to model $z$-dependent graded-index media. The focus- 
ing profiles (curvatures) of these waveguides can be made arbitrarily weak, so that even at optical frequencies the modes may be large and easily observed. In this study we have emphasized the experimental performance of metal waveguide models for tapered quadratic index media, which otherwise are not easily susceptible to experimental study.

The basic formulas governing the modes of curved metallic waveguides and of quadratic index waveguides are reviewed in Sec. II. Similarities and differences between these formulations are noted, and it is shown that for some of the mode properties the two waveguide types are mathematically equivalent. Section III includes experimental data for tapered metallic waveguides. Within experimental uncertainties the data are in agreement with recent theoretical models.

\section{Theory}

The theoretical formalism for treating the propagation of paraxial Hermite-Gaussian beams in quadratic graded index media has been well known for many years, so detailed derivations are omitted here. For the simplest slab geometry graded index material the square of the propagation coefficient can be written

$$
k^{2}(x, z)=k_{0}(z)\left[k_{0}(z)-k_{2}(z) x^{2}\right],
$$

where the wave will be assumed to propagate primarily in the $z$ direction. The complex amplitude of the linearly polarized electric field may be factored into a nearly plane wave with a slowly varying amplitude by means of the substitution

$$
E^{\prime}(x, z)=A(x, z) \exp \left[-i \int k_{0}(z) d z\right] .
$$

The resulting paraxial wave equation may be reduced exactly to a set of ordinary differential equations by assuming the Gaussian beam form ${ }^{2}$

$$
A(x, z)=\exp -i\left[Q(z) x^{2} / 2+S(z) x+P(z)\right] .
$$

The remaining equations are

$$
\begin{aligned}
Q^{2}+k_{0} \frac{d Q}{d z}+k_{0} k_{2} & =0, \\
Q S+k_{0} \frac{d S}{d z} & =0, \\
\frac{d P}{d z} & =-i \frac{Q}{2 k_{0}}-\frac{S^{2}}{2 k_{0}}-\frac{i}{2 k_{0}} \frac{d k_{0}}{d z} .
\end{aligned}
$$

The complex parameter $Q$ appearing in Eqs. (4)-(6) governs the transverse properties of the beam. It is conventional to introduce the complex beam radius $q$ $=k_{0} / Q$, and from Eq. (3) this complex radius has the form

$$
\frac{1}{q}=\frac{Q}{k_{0}}=\frac{1}{R_{p}}-\frac{i \lambda_{m}}{\pi w^{2}},
$$

where $R_{p}$ is the radius of curvature of the spherical phase fronts regarded as positive when the center of curvature is behind the phase front, $w$ is the $1 / e$ amplitude spot size, and $\lambda_{m}$ is the wavelength in the medium.
The complex parameter $S$ governs the transverse displacement of the beam. If the beam parameters are separated into their real and imaginary parts according to the formulas $Q=Q_{r}+i Q_{i}$ and $S=S_{r}+i S_{i}$, with Eq. (3) one can recognize $d_{a}=-S_{i} / Q_{i}$ as the displacement in the $x$ direction of the center of the amplitude distribution and $d_{p}=-S_{r} / Q_{r}$ as the displacement in the $x$ direction of the center of the phase fronts. The location and propagation direction of the beam with respect to the $z$ axis are thus determined by the real numbers $d_{a}$ and $d_{p}$.

The propagation characteristics of the fundamental off-axis Gaussian mode in a quadratic index medium can now be obtained by solving Eqs. (4)-(6). The solution of these equations for media in which $k_{0}$ and $k_{2}$ are constant has been known for many years, and several numerical and analytical techniques have recently been discussed for $z$-dependent or tapered media. ${ }^{2-4}$ While the fundamental mode is usually emphasized, there is not much extra difficulty in treating the higher order polynomial Gaussian modes.

Our principal interest here is in modeling graded index media with concave metal strip waveguides, so it is now appropriate to review the formulas governing the modes of such guides. It is an important and wellknown fact that the modes of these guides are in some respects identical to the modes of conventional graded index fibers. Thus the field distribution across the waveguide can be described in terms of off-axis Hermite-Gaussian functions. On the other hand, the field distribution perpendicular to the waveguide surface is most easily described in terms of Airy functions. The parameters governing the spot size and displacement of the Hermite-Gaussian transverse mode functions must satisfy the equations ${ }^{14}$

$$
\begin{aligned}
Q^{2}+k_{0} \frac{d Q}{d z}+\frac{k_{0}^{2}}{r R} & =0, \\
Q S+k_{0} \frac{d S}{d z} & =0,
\end{aligned}
$$

where the variable $z$ measures distance along the waveguide. The parameter $r$ is the radius of curvature of the waveguide along the direction of propagation, while $R$ is the radius of the concave waveguide transverse to the direction of propagation. In a tapered metal waveguide both $r$ and $R$ might be functions of $z$, and such a configuration is sketched in Fig. 1.

The most notable feature of Eqs. (8) and (9) is that they are identical in form to Eqs. (4) and (5) if one makes the identification

$$
\frac{k_{2}}{k_{0}}=\frac{1}{r R} .
$$

The propagation characteristics of graded index fibers are usually referred to the index of refraction

$$
n=n_{0}-n_{2} x^{2} / 2
$$

In terms of this index profile Eq. (10) is simply

$$
\frac{n_{2}}{n_{0}}=\frac{1}{r R} \text {. }
$$




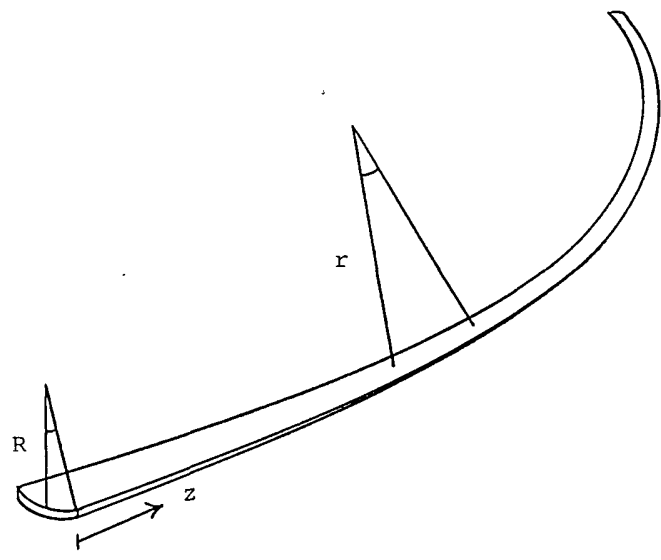

Fig. 1. Schematic representation of a concave metallic waveguide in which the curvature $r$ is a function of the propagation distance $z$, while the concaveness curvature $R$ is constant.

The significance of this result is that beam propagation in graded index media can be rigorously modeled using metal waveguides. This fact is especially useful with tapered media which are relatively difficult to analyze and fabricate.

\section{Experiment}

We carried out a series of experiments to confirm the theoretical results described in the previous section. These experiments involved a variety of waveguide materials and configurations. For most purposes it proved simplest to obtain the basic waveguide medium by sawing the side from a copper or aluminum tube. This rough waveguide can then be polished and bent as necessary. It is clear from Eq. (12) that a tapered graded index fiber corresponds to a metal waveguide in which either the waveguide curvature $r$ or the concaveness $R$ are functions of the propagation distance $z$. In the waveguides that we studied the curvature $r$ was chosen to be $z$ dependent.

The analogy between tapered graded index fibers and curved metal waveguides is valid for any $z$ dependence of the quadratic index coefficients. To be specific, we will describe here our experiments which tested the theoretical predictions using the $z$ dependence:

$$
\frac{n_{2}(z)}{n_{0}}=\frac{F}{(1-\gamma z)^{2}} .
$$

This functional form has often been considered to provide a realistic representation of practical fiber tapers. Although most of our theoretical solutions have been obtained numerically, it may be noted that Eq. (13) is also one of the few forms for which the solutions of Eqs. (4)-(6) can be expressed in terms of elementary functions. With Eq. (12) it follows that to model this profile the waveguide curvature should have the form

$$
r(z)=(1-\gamma z)^{2} / F R .
$$

For this curvature function the complex parameters $Q$ and $S$ from Eqs. (8) and (9) are given explicitly by the transformations

$$
\begin{gathered}
\frac{Q(z)}{k_{0}}=\frac{C(z)+D(z) Q\left(z_{1}\right) / k_{0}}{A(z)+B(z) Q\left(z_{1}\right) / k_{0}}, \\
S(z)=\frac{S\left(z_{1}\right)}{A(z)+B(z) Q\left(z_{1}\right) / k_{0}},
\end{gathered}
$$

where the $z$-dependent matrix elements are ${ }^{3}$

$$
\begin{aligned}
A= & (1-\gamma z)^{1 / 2}\left\{\cos \left[-\left(F-\gamma^{2} / 4\right)^{1 / 2} \gamma^{-1} \ln (1-\gamma z)\right]\right. \\
& \left.+\left(F-\gamma^{2} / 4\right)^{-1 / 2}(\gamma / 2) \sin \left[-\left(F-\gamma^{2} / 4\right)^{1 / 2} \gamma^{-1} \ln (1-\gamma z)\right]\right\} \\
B= & (1-\gamma z)^{1 / 2}\left(F-\gamma^{2} / 4\right)^{-1 / 2} \sin \left[-\left(F-\gamma^{2} / 4\right)^{1 / 2} \gamma^{-1} \ln (1-\gamma z)\right] \\
C= & -(1-\gamma z)^{-1 / 2} F\left(F-\gamma^{2} / 4\right)^{-1 / 2} \sin \left[-\left(F-\gamma^{2} / 4\right)^{1 / 2} \gamma^{-1} \ln (1-\gamma z)\right] \\
D= & (1-\gamma z)^{-1}\left\{\cos \left[-\left(F-\gamma^{2} / 4\right)^{1 / 2} \gamma^{-1} \ln (1-\gamma z)\right]\right. \\
& \left.-\left(F-\gamma^{2} / 4\right)^{-1 / 2}(\gamma / 2) \sin \left[-\left(F-\gamma^{2} / 4\right)^{1 / 2} \gamma^{-1} \ln (1-\gamma z)\right]\right\}
\end{aligned}
$$

Using these results one can write explicit expressions for the beam spot size $w(z)$ and amplitude displacement $d_{a}(z)$.

Several experimental tests of the theory have been carried out for waveguide curvatures given by Eq. (14). As an example, theoretical and experimental data are summarized in Fig. 2 for a 6328 - $\AA$ laser beam propagating along a waveguide made from a copper pipe having an inner diameter of $2 R=1.9 \mathrm{~cm}(0.75 \mathrm{in}$.). In practice, the curvature $r(z)$ was obtained by bending the waveguide to match a plywood template while $R$ was kept constant. The theoretical curve in this figure is a plot of the beam displacement implied by Eqs. (8) and (9) using the waveguide curvature given in Eq. (14). The parameter values used in Eq. (14) are $F=105 \mathrm{~m}^{-2}$ and $\gamma=0.5 \mathrm{~m}^{-1}$. The vertical lines in this figure mark the positions at which the maximum beam displacement was observed to occur when the beam was inserted parallel to the waveguide axis. The close agreement between experimental and theoretical data confirms the basic validity of the corresponding analysis.

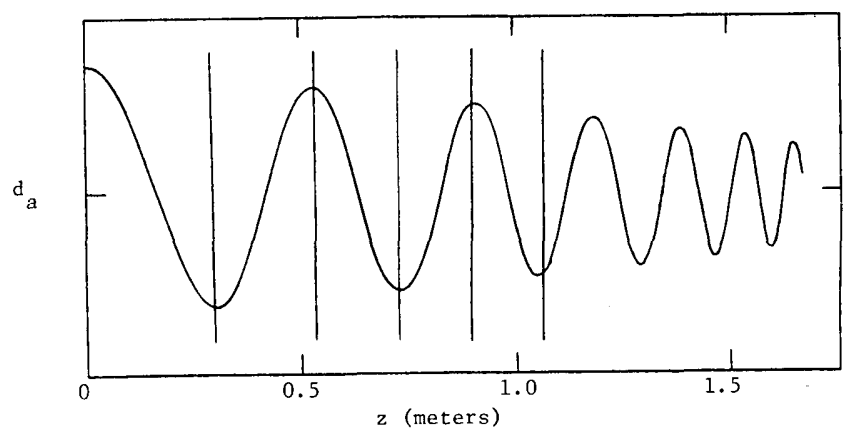

Fig. 2. Theoretical plot of the displacement of a Gaussian beam away from the axis of a tapered metal waveguide governed by Eq. (14) with the parameters $R=9.5 \mathrm{~mm}, F=105 \mathrm{~m}^{-2}$, and $\gamma=0.5 \mathrm{~m}^{-1}$. The vertical lines mark the experimentally observed positions of maximum beam displacement. 


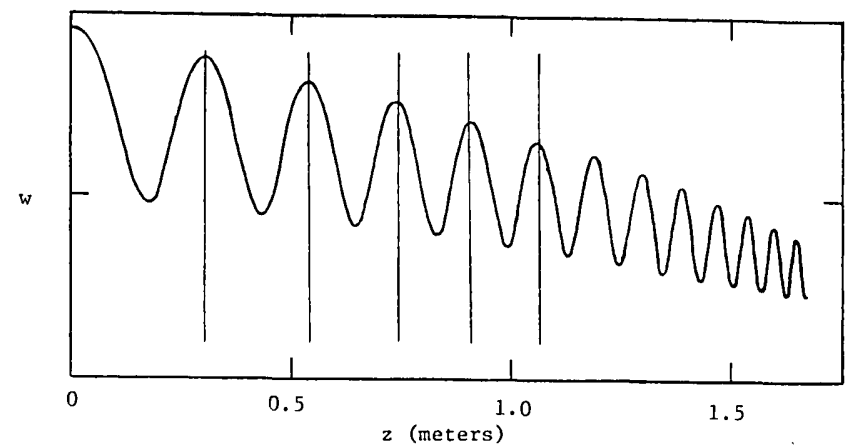

Fig. 3. Theoretical plot of the normalized spot size of a mismatched Gaussian beam in the same tapered metal waveguide represented in Fig. 2. The vertical lines mark the experimentally observed positions of maximum spot size.

The $z$ dependence of the beam spot size was also measured experimentally, and a typical result is shown in Fig. 3 for the same waveguide as in Fig. 2. The theoretical curve is the spot size implied by Eq. (7), and the vertical lines indicate the experimentally observed positions of maximum spot size. Other measurements of the mode functions can also be made, but for our purposes it has been sufficient to determine just the positions of maximum beam displacement and the positions of maximum and minimum spot size. Within the small experimental uncertainties these quantities are in agreement with theoretical expectations.

\section{Conclusion}

There have been many theoretical studies of beam propagation in graded index media, and in some cases the results apply to tapered media in which the parameters of the quadratic profile are functions of distance. Experimental studies of tapered media are, however, complicated by fabricational and measurement difficulties associated with the very small media, and only a few qualitative experiments have been reported. Since tapered graded index media may have many practical applications, it has seemed worthwhile to verify experimentally the theoretical results concerning tapered metal waveguides, which in important respects are equivalent to quadratic index waveguides. The experiments described here have confirmed that the spot size and displacement of off-axis beams in tapered metal waveguides behave as predicted by the theory.

This work was supported in part by the National Science Foundation and Tektronix, Inc.

\section{References}

1. A. Fletcher, T. Murphy, and A. Young, "Solutions of Two Optical Problems," Proc. R. Soc. London Ser. A 223, 216 (1954).

2. L. W. Casperson and J. L. Kirkwood, "Beam Propagation in Tapered Quadratic Index Waveguides: Numerical Solutions," IEEE/OSA Trans. Lightwave Technol. LT-3, 256 (1985).

3. L. W. Casperson, "Beam Propagation in Tapered Quadratic Index Waveguides: Analytical Solutions," IEEE/OSA Trans. Lightwave Technol. LT-3, 264 (1985).
4. L. W. Casperson, "Beam Propagation in Periodic QuadraticIndex Waveguides," Appl. Opt. 24, 4395 (1985).

5. S. Sheem and J. R. Whinnery, "Guiding by Single Curved Boundaries in Integrated Optics," Wave Electron. 1, 61 (1974).

6. M. Heiblum and J. H. Harris, "Analysis of Curved Optical Waveguides by Conformal Transformation,” IEEE J. Quantum Electron. QE-11, 75 (1975).

7. S. K. Sheem, "Three-dimensional Curved Surface for Integrated Optics," Appl. Opt. 14, 1854 (1975).

8. J. A. Arnaud, Beam and Fiber Optics (Academic, New York, 1976), pp. 16-19.

9. H. Krammer, "Light Waves Guided by a Single Curved Metallic Surface," Appl. Opt. 17, 316 (1978).

10. Lord Rayleigh (J. W. Strutt), "The Problem of the Whispering Gallery," Philos. Mag. 20, 1001 (1910).

11. Lord Rayleigh (J. W. Strutt), "Further Applications of Bessel's Functions of High Order to the Whispering Gallery and Allied Problems," Philos. Mag 27, 100 (1914).

12. M. E. Marhic, L. I. Kwan, and M. Epstein, "Optical Surface Waves along a Toroidal Metallic Guide,” Appl. Phys. Lett. 33, 609 (1 Oct. 1978).

13. M. E. Marhic, L. I. Kwan, and M. Epstein, "Invariant Properties of Helical-Circular Metallic Waveguides," Appl. Phys. Lett. 33, 874 (15 Nov. 1978).

14. L. W. Casperson and T. S. Garfield, "Guided Beams in Concave Metallic Waveguides,” IEEE J. Quantum Electron. QE-15, 491 (1979).

15. M. E. Marhic, "Polarization and Losses of Whispering-Gallery Waves Along Twisted Trajectories," J. Opt. Soc. Am. 69, 1218 (1979).

16. L. A. Wainstein, High-Power Electron. 3, 176 (1964).

17. G. Goubau and F. Schwering, "Modes in Radial Wave Beam Resonators," IEEE Trans. Microwave Theory Tech. MTT-13, 749 (1965).

18. L. W. Casperson, "Cylindrical Laser Resonators," J. Opt. Soc. Am. 63, 25 (1973).

19. L. W. Casperson and C. Romero, "Properties of a Radial Mode $\mathrm{CO}_{2}$ Laser," IEEE J. Quantum Electron. QE-9, 484 (1973).

20. M. E. Marhic, L. I. Kwan, and M. Epstein, "Whispering-Gallery $\mathrm{CO}_{2}$ Laser,” IEEE J. Quantum Electron. QE-15, 487 (1979).

21. J. G. Grossman, L. W. Casperson, and O. M. Stafsudd, "RadioFrequency-Excited Carbon Dioxide Metal Waveguide Laser," Appl. Opt. 22, 1298 (1983).

22. J. G. Grossman, L. W. Casperson, O. M. Stafsudd, and L. V. Sutter, Jr., "Propagation of Airy-Hermite-Gaussian Modes in Free Space," Appl. Opt. 23, 48 (1984). 